\title{
Cerebrospinal fluid HIV-1 p24 antigen and culture: sensitivity and specificity for AIDS-dementia complex
}

\author{
B J Brew, M O Paul, G Nakajima, A Khan, H Gallardo, R W Price
}

\begin{abstract}
We prospectively evaluated 94 patients with AIDS-dementia complex (ADC) and a smaller group of 27 patients with other HIV-1 related neurological conditions to determine the usefulness of cerebrospinal fluid (CSF) p24 antigen and HIV-1 culture in the diagnosis of ADC. The presence of ADC correlated with detectable CSF p24, but not with a positive culture. However, only $54 \%$ of the patients with severe or end stage (stages 3 and 4) ADC had detectable CSF p24 and only $25 \%$ had a positive culture. Among those with detectable CSF p24, there was no discernible relationship between the severity of ADC and the amount of CSF p24. The diagnostic sensitivity of CSF p24 in ADC was $21 \%$ whereas the specificity was $\mathbf{9 8 \%}$. CSF HIV-1 culture had a sensitivity of $30 \%$ and a specificity of $80 \%$. To address the possibility of binding of p24 in immune complexes, thereby escaping detection, an acid hydrolysis procedure was performed on the CSF and serum samples. This did not, however, make an appreciable difference in the detection rate of p24. To delineate whether the finding of cell free virus in the CSF was associated with ADC, CSF culture for HIV-1 was performed on both cell depleted and cell associated fractions. It was uncommon for CSF to be culture positive in only the cell free fraction and there was no relationship to the presence or severity of ADC.
\end{abstract}

$(\mathcal{F}$ Neurol Neurosurg Psychiatry 1994;57:784-789)

Infection with human immunodeficiency virus type 1 (HIV-1) may lead to a number of neurological complications, ${ }^{1}$ one of the most important of which is AIDS-dementia complex (ADC). In an appropriate patient, the diagnosis of $\mathrm{ADC}$ is made by the presence of characteristic symptoms and signs, and the exclusion of other causes of cognitive, motor, and behavioural deficit. Although the diagnosis of ADC is ultimately clinical, a specific and sensitive test for ADC would be exceptionally useful. Search for such a test has to date focused on the presence of the core antigen of HIV-1 in the cerebrospinal fluid (CSF), namely p24, and the ability to culture HIV-1 from the CSF.

Detection of p24 in the CSF of HIV-1 infected individuals has been associated with
HIV-1 related neurological disturbance in a number of studies. ${ }^{23}$ Furthermore, it has been shown that detection of p24 in the CSF is uncommon in asymptomatic patients. ${ }^{45}$ However, Portegies et $a l^{6}$ have recently reported that CSF p24 is only found in about half of the patients with ADC, thereby calling into question its diagnostic usefulness. Moreover, the relationship between the amount of p24 in the CSF and ADC severity has not been examined; the possibility that CSF p24 is undetectable because of immune complexing has also not been examined.

Although the diagnostic role of culture isolation of HIV-1 from the CSF also appears limited, because the virus has been isolated from the CSF of both asymptomatic and demented patients, ${ }^{7-12}$ there is some evidence from a small series of patients that culture positivity for cell free virus is associated with neurological disturbance. ${ }^{8}$

To define these issues more precisely, we examined HIV-1 p24 antigen and virus culture in both CSF and serum as a function of both the presence and severity of $\mathrm{ADC}$. The specificity of detectable CSF p24, and a positive culture from the CSF in the diagnosis of ADC, were determined by examination of their presence in patients with other neurological complications of HIV-1 infection. The hypothesis that detection of p24 was in part, confounded by the presence of p24 bound within immune complexes was addressed. Then, we examined the possibility that CSF culture positivity of cell free virus was associated with ADC. Last of all, the effect of Zidovudine on CSF p24 and culture positivity in the CSF was examined in a small subset of patients.

Patients and methods

Over a 19 month period, $142 \mathrm{CSF}$ and matching blood specimens were collected from $121 \mathrm{HIV}-1$ infected patients. Inclusion in this study was based on performance of a clinically indicated diagnostic lumbar puncture. All patients were seen by a neurologist and additional diagnostic investigations were carried out according to the clinical setting.

\section{PATIENT GROUPS}

On the basis of clinical evaluation and investigations, patients were assigned to one of two groups: the $\mathrm{ADC}$ group (94 patients, 108 samples) and the group with other HIV-1 related neurological complications (27 patients, 34 samples). 
The patients in the $\mathrm{ADC}$ group were diagnosed as having ADC if abnormal neurological symptoms and signs, and characteristic neuroimaging findings, were present ${ }^{1}$ and they had an ADC stage of $\geqslant 1$. (Severity was rated on a functionally based scale ranging from 0 to $4^{13}$ : stage 0 (normal), stage 0.5 (equivocally abnormal symptoms or signs), stage 1 (mild), stage 2 (moderate), stage 3 (severe), stage 4 (very severe).) Stage 0 and 0.5 patients were included in this group to assist in the determination of the usefulness of CSF antigen and culture in the diagnosis of ADC. Such patients were evaluated because of the presence of equivocal cognitive complaints or headache. As there was only one patient with stage 0 , this was combined with stage 0.5 for analysis. Similarly, because of the small numbers of patients with stage 4 disease, these were combined with stage 3 for analysis. The number of patients and the number of samples for each of these stages are as follows: Stages 0-0.5: 38 patients and 41 samples; stage 1: 27 patients and 34 samples; stage 2: 18 patients and 22 samples; stages 3 and 4: 11 patients and 11 samples. Thirty nine of the 94 patients were taking zidovudine at the time of assessment, and 10 patients had CSF and blood available immediately before starting zidovudine and after an average of eight weeks on zidovudine.

The group with other HIV-1 related neurological complications consisted of the following: primary central nervous system lymphoma (seven patients, eight samples), aseptic meningitis (six patients, ten samples), cryptococcal meningitis (five patients, six samples), herpes zoster myelitis (two patients, three samples), cerebral toxoplasmosis (two patients, two samples), progressive multifocal leucoencephalopathy (two patients, two samples), cytomegalovirus radiculomyelitis (two patients, two samples), and cerebrovascular disease (one patient, one sample).

\section{LABORATORY METHODS}

CSF cell count, protein, glucose, cryptococcal antigen, cytology, and Venereal Disease Reference Laboratory test were obtained along with bacterial and fungal cultures. To determine the integrity of the blood-brain barrier, CSF and serum albumin levels were collected. A value of $\leqslant 6.5$ for the CSF: serum albumin ratio $\times 1000$ was considered abnormal. ${ }^{14}$

\section{Detection of HIV-1 p24}

The presence of HIV-1 antigen was determined in CSF and serum samples by a solid phase immunoassay, carried out according to the manufacturer's instructions (Abbott Laboratories, Chicago, Illinois, USA). The threshold for detection of $\mathrm{p} 24$ was found to be $30 \mathrm{pg} / \mathrm{ml}$.

This test may uncommonly $(<10 \%$ of the time) cross react with other HIV-1 antigens, such as p18, p55, gp41, gp120, and gp160. As a result of this and the consequent increased detection rate for the assay, the HIV-1 antigen content in 67 cell culture supernatants, obtained from inoculated peripheral blood leucocytes and macrophage cultures, was examined concurrently by both the Dupont (p24) and Abbott systems used in this study. There was a $100 \%$ concordance between the two systems with regard to positive and negative samples. Therefore, we used the Abbott HIV-1 p24 ELISA (enzyme-linked immunosorbent assay) kit to determine the presence of HIV-1 p24.

To determine whether detection of $\mathrm{p} 24$ was influenced by the presence of immune complexes, and acid treatment procedure as described by Mathiesen et $a l^{15}$ was performed on a subset of the patients: 82 samples of CSF and 10 of sera. Acid hydrolysed samples were evaluated at the same time as the same untreated specimens and tested by an HIV-1 p24 ELISA kit (Abbott Laboratories, Chicago, Illinois, USA).

\section{HIV-1 culture}

Methods used were essentially those described by $\mathrm{Ho}$ et al. ${ }^{10}$ Briefly, peripheral blood mononuclear cells from the buffy coats of blood donors, who were seronegative for HIV-1 antibody and hepatitis B surface antigen, were separated by centrifugation in Ficoll-Paque (Pharmacia, Piscataway, New Jersey, USA). The cells were washed twice with phosphate buffered saline (PBS) before being stimulated at $37^{\circ} \mathrm{C}$ for three days with phytohaemagglutinin A (Difco Laboratories, Detroit, Michigan, USA) in RPMI medium with hydroxyethylpiperazine-ethane sulphuric acid (HEPES) buffer, supplemented with $20 \%$ fetal calf serum (FCS) and antibiotics (100 units penicillin and $100 \mu \mathrm{g}$ streptomycin-Biofluids, Rockville, Maryland, USA). Stimulated cells were washed twice with PBS, divided into aliquots of $10^{6} \mathrm{cells} / \mathrm{ml}$ and stored at $-70^{\circ} \mathrm{C}$ in $10 \%$ dimethyl sulphoxide (DMSO) in interleukin-2 (IL-2) medium (RPMI with HEPES, supplemented with $20 \%$ FCS, 5\% IL-2 (Advanced Biotechnologies Inc., Columbia, Maryland, USA)), $2 \mu \mathrm{g} / \mathrm{ml}$ polybrene (Sigma Chemical Company, St Louis, Missouri, USA) and antibiotics. These indicator lymphocytes were rapidly thawed (at $37^{\circ} \mathrm{C}$ ), washed once with IL-2 medium and seeded in $5 \mathrm{ml} \mathrm{IL-2}$ medium at $5 \times 10^{6} /$ per $25 \mathrm{~cm}^{3}$ polystyrene tissue culture flask (Corning Glass Works, Corning, New York, USA).

Freshly thawed lymphocytes in IL-2 medium were inoculated with $0.5 \mathrm{ml} / 25 \mathrm{~cm}^{3}$ flask for each CSF sample and for each patient's lymphocytes in PBS, which was separated as above with Ficoll-Paque but without stimulation.

Flasks were incubated at $37^{\circ} \mathrm{C}$ and supernatants of cultures tested weekly for HIV-1 p24 antigen by an immunoassay (Dupont, New England Nuclear Research Products, Billerica, Maryland, USA). HIV-1 p24 antigen is captured by the highly specific rabbit polyclonal antibody fixed to microtitre wells. Captured HIV-1 p24 core antigen was then complexed with biotinylated polyclonal antibodies to HIV-1 p24 core antigen and 
probed with a streptavidin-HRP (horse radish conjugate). The complex was detected by incubation with $o$-phenyldiamine hydrochloride which produced a yellow colour directly proportional to the amount of p24 antigen captured. The absorbance of each well was determined using a microplate reader and calibrated against the absorbance of an HIV-1 p24 antigen standard curve.

Cultures were kept for four weeks and only those that were consecutively positive for two weeks were considered to be HIV-1 positive.

In a subset of samples, low speed centrifugation was used to collect the cells from the CSF, the cell depleted CSF was then centrifuged at $4500 \mathrm{rpm}$ for 30 minutes to concentrate free virus further. This was then used to inoculate the freshly prepared lymphocytes as described above.

\section{STATISTICS}

Statistical methods included analyses of variance and Student-Newman-Keuls (SNK) post hoc tests in comparisons of discrete disease groups and ADC stages. Relationships among continuous variables were assessed using Pearson correlations. The evaluations of independence among variables were conducted with $\chi^{2}$ tests. Alpha significance level was established at $\mathrm{p} \leqslant 0.05$. The patients with multiple CSF and blood analyses were included in the main data set as independent observations because the CSF and blood were repeated for assessment of change in clinical condition. Analyses were carried out using SPSS/PC + software (SPSS Inc., Chicago, Illinois, USA).

\section{Results}

HIV-1 p24

In the ADC group, 15 of 108 (14\%) CSF samples and 55 of 101 (54\%) serum samples were positive for HIV-1 p24 antigen. The frequency of positive antigen increased progressively with the stage of ADC in both CSF and serum specimens; only $2 \%$ of CSF specimens

Figure 1 Relationship between percentage of samples with detectable HIV-1 p24 (filled bars) and positive culture isolation of HIV-1 (open bars) in (A) CSF and (B) blood in relation to $A D C$ stage in the $A D C$ patient group.

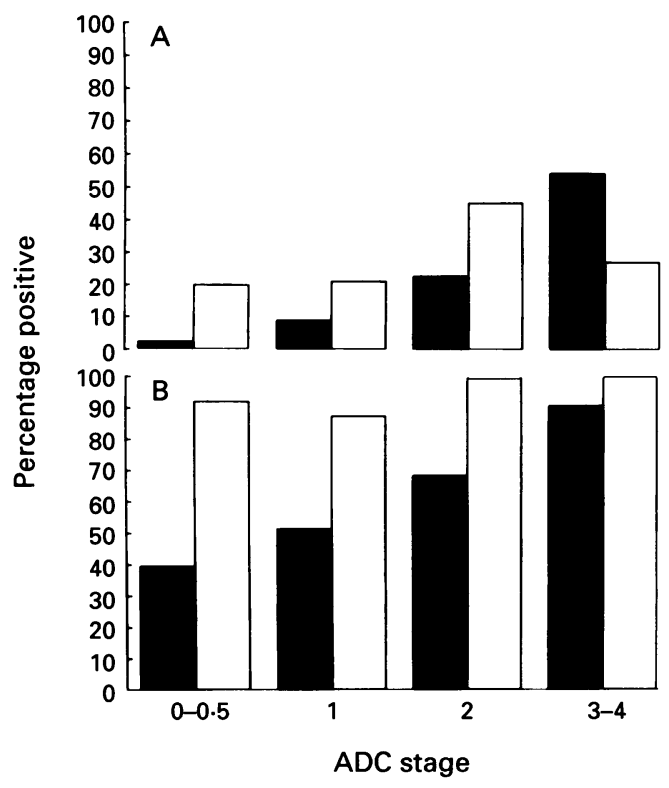

in stage 0 or 0.5 were positive, whereas $54 \%$ of specimens in stage 3 or 4 were positive (fig $1 \mathrm{a}, 1 \mathrm{~b})$. The presence of $\mathrm{p} 24$ in the CSF but not in the serum correlated with ADC severity $(r=0.52, \mathrm{p}<0.01 ; r=0.11$ respectively).

Within each ADC stage, the p24 antigen level varied considerably, and the level in the serum was uniformly much higher than in the CSF. The quantity of $\mathrm{p} 24$ in neither the CSF nor the serum correlated with $\mathrm{ADC}$ severity ( $r=0.34$ and 0.01 respectively). The concentration varied from 0 to $12800 \mathrm{pg} / \mathrm{ml}$ for sera and from 0 to $731 \mathrm{pg} / \mathrm{ml}$ for CSF. A one way analysis of variance for CSF $(\mathrm{pg} / \mathrm{ml})$ values of p24 antigen showed an overall difference among the four ADC stages $(F=6 \cdot 8$; df $=$ $3104 ; \mathrm{p}<0.0005)$ and the Student-NewmanKeuls procedure $(p<0.05)$ revealed that stages 2 and 3 were statistically different from stages 0 and 1 . The corresponding analysis for the serum $(\mathrm{pg} / \mathrm{ml})$ values did not show a difference between the ADC stages $(F=0 \cdot 85, \mathrm{df}$ $=3112$ ), whereas the analysis for the presence of detectable p24 in the serum, rather than quantity, showed a difference according to stage $(F=6.34, \mathrm{df}=3115, \mathrm{p}<0.0001)$ with stages 3 and 4 being different from stage 1. The mean and standard deviation for the samples which were positive for p24 antigen in CSF and serum are shown in table 1.

In patients with other HIV-1 related neurological conditions, p24 was found in the CSF in only three instances: one patient each with aseptic meningitis, herpes zoster myelitis, and CMV radiculomyelitis. Precise characterisation of the presence of concomitant ADC in these patients was only possible in the patient with aseptic meningitis in whom the $\mathrm{ADC}$ stage was considered to be 1 .

Eighteen of the $80 \mathrm{CSF}$ samples and one of the 10 serum samples were found to be positive after the acid treatment procedure. Six of the $18 \mathrm{CSF}$ samples became positive only after treatment, whereas, conversely, two patients' CSF became negative after treatment, previously having been positive. However, there was no significant difference between the amount of antigen in the untreated versus that in the treated group ( $t=$ $1.08, \mathrm{df}=81, \mathrm{p}=0.28)$ and the increase in p24 was small. In the samples that became positive, the mean of the difference between untreated and treated p24 values was 6.67 (SEM 4.34).

HIV-1 CULTURE

HIV-1 was isolated from $26 \%$ of CSF samples

Table 1 Amount of $p 24$ detected in positive samples of $C S F$ and blood of $A D C$ group

\begin{tabular}{|c|c|c|c|c|c|c|}
\hline \multirow{3}{*}{$\begin{array}{l}A D C \\
\text { stage }\end{array}$} & \multicolumn{6}{|c|}{$p 24(p g / m l)$ in } \\
\hline & \multicolumn{3}{|c|}{$C S F$} & \multicolumn{3}{|c|}{ Serum } \\
\hline & No. & Mean & $S D$ & No. & Mean & $S D$ \\
\hline $\begin{array}{l}0-0 \cdot 5 \\
1 \\
2 \\
3-4\end{array}$ & $\begin{array}{l}1 \\
3 \\
5 \\
6\end{array}$ & $\begin{array}{r}53 \\
50 \\
335 \\
212\end{array}$ & $\begin{array}{r}0 \\
11 \\
243 \\
182\end{array}$ & $\begin{array}{l}15 \\
17 \\
13 \\
10\end{array}$ & $\begin{array}{r}1919 \\
1447 \\
557 \\
1503\end{array}$ & $\begin{array}{r}3258 \\
2833 \\
571 \\
1944\end{array}$ \\
\hline Total & 15 & 210 & 204 & 55 & 1376 & 2466 \\
\hline
\end{tabular}


and $93 \%$ of blood samples (fig 1) from patients with ADC. The severity of ADC did not correlate with a positive culture in either the CSF or blood ( $r=0 \cdot 10$ and $0 \cdot 13$ respectively). Similarly, there was no significant relationship between the presence of $\mathrm{ADC}$ and the positivity of the CSF culture $\left(\chi^{2}=5 \cdot 4\right.$; $\mathrm{p}<0.15)$ or in blood $\left(\chi^{2}=3.7 ; \mathrm{p}<0.30\right)$, probably resulting from the influence of the CSF cell count on culture and the fact that the cell count increased up to ADC stage 2 and then fell.

In patients with other HIV-1 related neurological diseases, approximately $50 \%$ of the CSF samples had positive cultures for HIV-1, with rates varying from $33 \%$ to $67 \%$ depending on the underlying disease. In the blood, $96 \%$ were culture positive with only one of the samples of a patient with aseptic meningitis being negative.

Of $80 \mathrm{CSF}$ samples cultured with both cell depleted and cell associated fluid, a total of 34 (43\%) were culture positive. There were seven $(9 \%)$ that were positive only in cell depleted fluid and $12(15 \%)$ only in cell associated fluid, whereas $15(19 \%)$ were positive in both. There was no relationship between culture positivity for cell depleted and cell associated fluids and ADC presence or severity.

\section{SPECIFICITY, SENSITIVITY, PREDICTIVE VALUE} AND EFFICIENCY

Table 2 presents a formal analysis of the diagnostic use of HIV-1 direct antigen test on CSF and serum, and of HIV-1 culture in CSF and blood for ADC. The specificity of CSF p24 is high (98\%) but the sensitivity is low (21\%). CSF culture was lower in specificity $(81 \%)$ and also in sensitivity (30\%). The overall efficiency was equivocal in both cases: $50 \%$ and $49 \%$ respectively.

\section{CSF CELL COUNT}

In an attempt to determine whether a low CSF cell count provided an explanation for those samples of CSF from which HIV-1 could not be cultured in spite of the presence of detectable p24, analyses of the CSF cell count by ADC stage and culture results were performed. There was a pronounced variability of CSF cell count in relation to ADC stage. The mean count was 2.8 for stage $0-0.5,1.6$ for stage $1,9.4$ for stage 2 , and 0.8 for stage 3-4. There was a significant relationship between the mean cell count and the CSF culture positivity $(p<0.005)$. This may account for the highest isolation rate occur-

Table 2 Analysis of the diagnostic use of p24 detection and HIV-1 isolation in CSF and blood

\begin{tabular}{|c|c|c|c|c|}
\hline & \multicolumn{2}{|l|}{$p 24$} & \multicolumn{2}{|c|}{ HIV-1 culture } \\
\hline & $C S F$ & Serum & $C S F$ & Blood \\
\hline $\begin{array}{l}\text { Sensitivity (\%) } \\
\text { Specificity (\%) } \\
\text { Predictive value of a positive test (\%) } \\
\text { Predictive value of a negative test (\%) } \\
\text { Efficiency (\%) }\end{array}$ & $\begin{array}{l}21 \\
98 \\
93 \\
43 \\
50\end{array}$ & $\begin{array}{l}63 \\
61 \\
72 \\
50 \\
62\end{array}$ & $\begin{array}{l}30 \\
80 \\
71 \\
41 \\
49\end{array}$ & $\begin{array}{r}93 \\
8 \\
62 \\
9 \\
61\end{array}$ \\
\hline
\end{tabular}

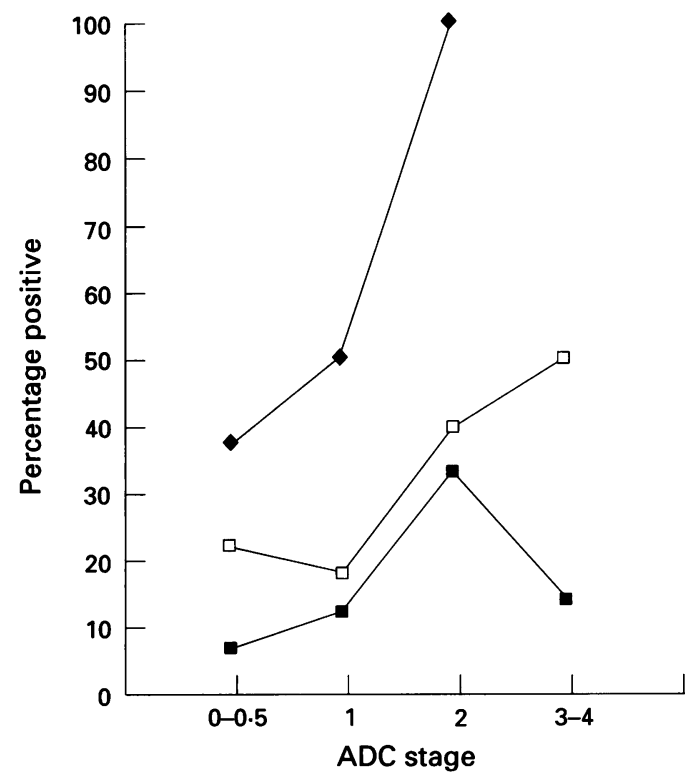

Figure 2 Relationship between percentage of samples with Figure 2 Relationship between percentage of samples with
positive HIV-1 culture in the CSF according to $A D C$ stage positive HIV-1 culture in the CSF according to ADC stage
for different CSF cell counts: $\square, 0$ cells; $\square, 1-3$ cells; $\diamond$, $\geqslant 4$ cells.

ring in stage 2 patients and the decline in stage 3-4 patients. The mean CSF cell count was 10.0 for patients with positive CSF cultures for HIV-1 and 1.3 for patients with negative cultures; this difference was significant $(F[1104]=8.3 ; \mathrm{p}<0.005)$.

Figure 2 shows that culture positivity was more frequent when the CSF cell count was increased.

\section{BLOOD-BRAIN BARRIER}

Forty two per cent of the samples from $\mathrm{ADC}$ patients had a disruption of the integrity of the blood-brain barrier to albumin. There was no relationship between ADC stage and the presence of an impairment of the blood-brain barrier $\left(\chi^{2}=3.7, p<0.3\right)$, and no correlation between the two as measured by the albumin ratio $(r=0.093, \mathrm{p}=0.57)$. In addition there was no relationship between the impairment of the blood-brain barrier and p24 in the CSF $\left(\chi^{2}=0.8, \mathrm{p}<0.36\right)$ or impairment and HIV-1 culture $\left(\chi^{2}=1 \cdot 2, \mathrm{p}<0 \cdot 26\right)$.

\section{EFFECT OF ZIDOVUDINE}

Of the 10 patients from whom samples of CSF and sera were available before and during treatment with zidovudine, there was only one CSF sample that was positive for p24, which became negative on therapy. Four CSF samples were positive for HIV-1 by culture and each of these became negative on treatment. However, one CSF sample that had previously been culture negative became positive on therapy.

\section{Discussion}

These results confirm that detection of CSF p24 is a relatively specific diagnostic technique although it is also insensitive to $\mathrm{ADC}$, whereas culture of HIV-1 from the CSF is even less specific. Thus, although overall the 
presence of CSF p24 correlated with the severity of ADC, antigen was not detected in most cases. Indeed, even though the correlation with $\mathrm{ADC}$ severity was not particularly strong, it is unlikely to merely reflect the advanced state of systemic disease in these patients because only half of them with dementia of stages 3 and 4 had detectable p24 in spite of their advanced systemic disease; also CSF p24 was only detectable in three of 27 patients with other HIV-1 related neurological conditions, or two of 20 who had illnesses diagnostic of AIDS. The infrequency of CSF p24 detection was only partly explained by the presence of immune complexes, at least as determined by the particular acid treatment method. Neither CSF p24 nor HIV-1 culture was related to the integrity of the blood-brain barrier. Culture positivity in the CSF was, however, in part related to the CSF cell count. Although the number of observations of the effect of zidovudine on p24 and culture was small, the results suggest that CSF p24 was not useful in monitoring response to zidovudine therapy because it was only rarely positive. Conversely, CSF culture usually became negative on treatment.

Other studies have also shown that p24 in the CSF has limited usefulness in the diagnosis of ADC. ${ }^{6}$ In our series, p24 detection was only achieved at a level approaching $50 \%$ in patients with ADC stages 3 and 4 . In such severely afflicted patients, clinical diagnosis is usually made easily and hence the practical value is usually limited in spite of a relatively specific finding. In those with milder disease, sensitivity of CSF antigen detection is even lower. Nevertheless, it may have some use as an ancillary aid in isolated patients. For practical diagnosis, other CSF markers, such as $\beta_{2}$ microglobulin and neopterin, ${ }^{16}{ }^{17}$ may be more useful. The increasing rate of blood antigen positivity with ADC severity is of interest in suggesting that this neurological condition parallels advancing systemic HIV-1 infection and viral replication. However, serum p24 detection is not useful for practical ADC diagnosis. Similarly, HIV-1 culture of either CSF or blood is not clinically useful for ADC diagnosis because of the non-specificity of positive cultures of either fluid.

Although the anatomical source of CSF p24 antigen is uncertain, the frequency of detection in relation to ADC severity is not dissimilar to our own necropsy experience ${ }^{18}$ and that of others ${ }^{19}$ in which milder ADC (stages 1 and 2) may exhibit limited or no immunohistochemically detectable p24. Such necropsy observations, along with CSF studies of markers of immune activation and p24 detection, are consistent with the hypothesis of a role for cytokines in the brain injury of ADC. ${ }^{20} 21$ Varying sources of p24 might explain the discrepancy between CSF antigen and culture results, if culture isolation depends principally on virus infection of CSF cells, whereas p24 might derive from either infected CSF cells or diffusion from infection of the brain parenchyma which might not be accompanied by similar movement of infec- tious virus. Relative macrophage tropism of brain variants of HIV-1 might also account in part for the discrepancy between p24 and culture results; our culture system used fresh peripheral blood mononuclear cells which have been shown to support replication of macrophage tropic variants. Quantitative differences in sensitivity of the culture system to evolving variants may, however, have made a difference. Careful characterisation of serial evolution of viral variants in CSF may help to resolve this issue.

In conclusion, CSF p24 is infrequently detectable in patients with ADC, but when it is found it may be useful as an aid to the diagnosis and response to therapy. Culture of HIV-1 from the CSF, on the other hand, is both nonspecific and less sensitive.

This study was supported by US Public Health Service Research Grants NS 25701 and a grant from the Rudin Foundation. We thank William Wolf for help in data management, and Howard Thaler for assistance with statistical analysis.

1 Brew BJ, Sidtis JJ, Petito CK, Price RW. The neurologic complications of AIDS and human immunodeficiency virus infection. In: Plum F, ed. Advances in contemporary neurology. Contemporary neurology series. Philadelphia: FA Davis \& Co 1988:1-49.

2 Goudsmit G, De Wolf F, Paul DA, et al. Expression of Human Immunodeficiency Virus antigen (HIV-Ag) in serum and cerebrospinal fluid during acute and chronic infection. Lancet 1986;ii: 177-80.

3 Epstein LG, Goudsmit J, Paul DA, et al. Expression of Human Immunodeficiency Virus in cerebrospinal fluid of children with progressive encephalopathy. Ann Neurol of children with progt

4 Marshall DW, Brey RI, Cahill WT, et al. Spectrum of CSF findings in various stages of HIV infection. Arch Neurol findings in various

5 McArthur JC, Cohen BA, Farzadegan H, et al. Cerebrospinal fluid abnormalities in homosexual men with and without neuropsychiatric findings. Ann Neurol 1988;23 (suppl):S34-7.

6 Portegies P, Epstein LG, Hung STA, de Gans J, Goudsmit J. Human Immunodeficiency Virus Type 1 antigen in cerebrospinal fluid correlation with clinical neurologic status. Arch Neurol 1989;46:261-4.

7 Chiodi F, Aasjoe B, Fenyoe EM, Norkrans G, Hagberg L, Albert J. Isolation of the human immunodeficiency virus from the cerebrospinal fluid of an antibody positive virus carrier without neurological symptoms. Lancet 1986;ii: carrier with $1276-7$.

8 Chiodi F, Sonnerborg A, Albert J, et al. Human immunodeficiency virus infection of the brain. I. Virus isolation and detection of HIV specific antibodies in the cereand detection of HIV specific antibodies in the cerebrospinal fluid of patients with varyin
tions. $\mathcal{F}$ Neurol Sci 1988;85:245-57.

9 Chiodi F, Albert J, Olausson E, et al. Isolation frequency of Human Immunodeficiency Virus from cerebrospinal fluid and blood of patients with varying severity of HIV infection. AIDS Res Hum Retroviruses 1988;4:351-8.

10 Ho DD, Rota TR, Schooley RT, et al. Isolation of HTLVIII from cerebrospinal fluid and neural tissue of patients with neurological syndromes related to the acquired immunodeficiency syndrome. $N$ Engl $f$ Med 1985;313: 1493-7.

11 Levy JA, Shimabukuro J, Hollander H, Mills J, Kaminsky L. Isolation of AIDS-associated retroviruses from cerebrospinal fluid and brain of patients with neurological brospinal fluid and brain of patient

12 Resnick L, Berger J, Shapshak P, Tourtellotte WW. Early Resnick L, Berger J, Shapshak P, Tourtellotte WW. Early
penetration of the blood-brain barrier by HIV. Neurology penetration of

13 Price RW, Brew BJ. The AIDS dementia complex. 7 Infect Dis 1988;158:1079-83.

14 Eeg-Olofsson O, Link H, Wigertz A. Concentrations of CSF proteins as a measure of blood brain barrier function and synthesis of IgG within the CNS in "normal" subjects from the age of 6 months to 30 years. Acta Paediatr Scand 1981;70:167-70.

15 Mathiesen T, Sundqvist VA, Albert J, Ohlsson E, Wahren $B$. Acid hydrolysis of serum samples to increase detection of HIV antigen. $\mathcal{F}$ Virol Methods 1988;22:143-8.

16 Brew BJ, Bhalla RB, Fleisher M, et al. Cerebrospinal fluid $\beta_{2}$ microglobulin in patients infected with human immunodeficiency virus. Neurology 1989;39:830-4.

17 Brew BJ, Bhalla RV, Paul M, et al. Cerebrospinal fluid neopterin in HIV-1 infection. Ann Neurol 1990;28: 556-60.

18 Cronin KC, Rosenblum M, Brew BJ, Price RW. HIV-1 brain infection: distribution of infection and clinical correlates. Fifth International AIDS Conference, correlates. Fifth International AIDS
Montrèal, June 1989 [abstract WCP 45]. 
19 Vazeux R, Cumont MC, Ciaudo C, et al. Severe HIVrelated encephalopathy in children without any HIV replication in the central nervous system. The neurological and neuropsychological complications of HIV infection, update 1990, Monterey 1990, [abstract NPS-15]

20 Price RW, Brew B, Sidtis J, Rosenblum M, Scheck AC, Cleary P. The brain in AIDS: central nervous system
HIV-1 infection and the AIDS dementia complex Science 1988;239:586-92.

21 Price RW, Brew BJ, Rosenblum M. The AIDS dementia complex and HIV-1 infection: a pathogenetic model of virus-immune interaction. In: Waksman $\mathrm{BH}$ ed, Immunologic mechanisms in neurologic and psychiatric disease. New York: Raven Press 1990.

\section{NEUROLOGY IN LITERATURE}

\section{Vasovagal attacks}

Thomas Lewis published his observations upon fainting attacks in $1918,{ }^{1}$ suggesting that "accurate observations upon the cardiovascular system during the faints of which young men and women of nervous disposition are the subjects have not as yet been obtained, or obtained have not been recorded." Such descriptions were available to Lewis, although not in the scientific literature. The following extracts include an early account by Diderot and analyses of the warning symptoms by Jane Austen, George Eliot, Wilkie Collins, and Arnold Bennett. Dostoyevsky mistakenly assumed the loss of posture to be precipitate rather than gradual. Dickens believed faints to be virtually a female prerogative-indeed of the twenty or so descriptions in his novels, all but one are female, the exception, in Oliver Twist, having had a previous event that suggested epilepsy was the more likely diagnosis. Dickens assumed that faints were either a response to a physical insult, or a deliberate manipulation on the part of the sufferer. The witnessing of blood-letting can be a potent trigger as Flaubert's account of a "double faint" testifies. Authors have also touched on the management of syncope in some cases with a touching belief in the efficacy of vinegar, but in others with awareness of the value of the horizontal posture. Cardiac syncope can be difficult to distinguish from vasovagal attacks, though in some cases, symptoms such as palpitations suggest the possibility, as with Captain Wybrow in Scenes of clerical life.

D Diderot, 1796, The nun

I moved towards the superior with my arms held out in supplication and my body leaning backwards, swooning. I fell, but it was not a heavy fall. In such fainting fits when one's strength abandons one, the limbs seem to give way and as it were fold up unawares; nature, unable to hold up, seems to try to collapse gently. I lost consciousness and the sense of feeling, and merely heard confused and distant voices buzzing round me; whether it was real speech or a singing in my ears, I could make out nothing but this continual buzzing.

fane Austen, 1811, Sense and Sensibility Marianne, now looking dreadfully white, and unable to stand, sunk onto her chair, and Elinor, expecting every moment to see her faint, tried to screen her from the observation of others, while reviving her with lavender water.

Charles Dickens, 1837-9, Oliver Twist

"... turned his hands in his hair; and, with a loud scream, rolled grovelling upon the floor, his eyes fixed, and the foam covering his lips."

Charles Dickens, 1839, Nicholas Nickleby

"Miss Petowker was at length supported in a condition of much exhaustion to the first floor, where she no sooner encountered the youthful bridegroom than she fainted with great decorum."

\section{Gustave Flaubert, 1856-7, Madame Bovary}

So Bovary sent for a bandage and basin, which he asked Justin to hold. "Don't be afraid, my man" he said to the already white-faced villager.

"No, no, go ahead!" the man answered. And he held out his brawny arm with a touch of bravado. At the prick of the lancet the blood spurted out and splashed against the mirror.
"Nearer with the basin!" Charles exclaimed. "Lookee!" said the peasant. "It's like a young fountain flowing! What red blood I've got. Should be a good sign, eh?"

"Sometimes", the Officer of Health remarked, "they don't feel anything at first: the syncope occurs afterwards, especially with strong chaps like this".

Instantly the yokel let go of the lancet-case which he had been twirling in his fingers: a jerk of his shoulders snapped the back of his chair: his cap fell to the floor.

"I thought as much", said Bovary, putting his fingers over the vein.

The basin began to wobble in Justin's hands. $\mathrm{He}$ quaked at the knees. His face went white.

"Emma!" Charles called out, "Emma!" She was down the stairs in a flash "Vinegar!" he cried. "Good Lord, two at once!"

George Eliot, 1858, Scenes of clerical life

By jove, what a rate my heart is galloping at! These confounded palpitations get worse instead of better... Still he might only have fainted; it might only be a fit.

Sir Christopher knelt down, unfastened the cravat, unfastened the waistcoat, and laid his hand on the heart. It might be syncope; it might not-it could not be death. No! that thought must be kept far off.

George Eliot, 1859, Adam Bede

"Look there! She's fainting" said the landlady, hastening to support Hetty, who had lost her miserable consciousness and looked like a beautiful corpse.

Wilkie Collins, 1861, The dead secret

She staggered desperately, a few paces further, and reached the first row of doors that opened on the landing. There nature sank exhausted: her knees gave way under her-her breath, her sight, her hearing all seemed to fail her together at the same instant-and she dropped down senseless on the floor at the head of the stairs.

Fedor Dostoyevsky, 1871, The devils

But she suddenly uttered a scream and fell full length on the floor in a faint. I can still hear the thud of her fall as her head hit the carpet.

Arnold Bennett, 1904, Teresa of Watling Street Never raise the head of a person who has lost consciousness, he said coldly, it is dangerous. Teresa will recover in a few minutes. This swoon is due only to the shock and strain of the last few minutes.

Arnold Bennett, 1911, Hilda Lessways

She felt dizzy ... the door of Sarah's wardrobe was ajar, and, in the mirror of it, Hilda could see herself obscurely, a black-robed strange young woman, with untidy hair and white cheeks and huge dark, staring heavy eyes, with pouches beneath them. The image wavered in the mirror. She thought: "Here it is again, this awful feeling! Surely I am not going to faint!?” G D PERKIN

Regional Neurosciences Centre, Charing Cross Hospital,
London W6 $8 R F$, UK

1 Cotton TF, Lewis T. Observations upon fainting attacks due to inhibitory cardiac impulses. Heart 1918-20;7: 23-4. 